\title{
A Simple, Efficient Synthesis of 2-Aryl Benzimidazoles Using Silica Supported Periodic Acid Catalyst and Evaluation of Anticancer Activity
}

\author{
Vyankat A. Sontakke, ${ }^{1}$ Sougata Ghosh, ${ }^{2}$ Pravin P. Lawande, ${ }^{1}$ \\ Balu A. Chopade, ${ }^{2}$ and Vaishali S. Shinde ${ }^{1}$ \\ ${ }^{1}$ Garware Research Centre, Department of Chemistry, University of Pune, Pune 411007, India \\ ${ }^{2}$ Institute of Bioinformatics and Biotechnology, University of Pune, Pune 411007, India
}

Correspondence should be addressed to Vaishali S. Shinde; vaishali.san@gmail.com

Received 14 March 2013; Accepted 3 April 2013

Academic Editors: D. K. Chand, G. Gattuso, and J. C. Menéndez

Copyright ( $) 2013$ Vyankat A. Sontakke et al. This is an open access article distributed under the Creative Commons Attribution License, which permits unrestricted use, distribution, and reproduction in any medium, provided the original work is properly cited.

A new, efficient method for the synthesis of 2-aryl substituted benzimidazole by using silica supported periodic acid $\left(\mathrm{H}_{5} \mathrm{IO}_{6}-\mathrm{SiO}_{2}\right)$ as a catalyst has been developed. The salient feature of the present method includes mild reaction condition, short reaction time, high yield and easy workup procedure. The synthesized benzimidazoles exhibited potent anticancer activity against MCF7 and HL60 cell lines.

\section{Introduction}

The benzimidazole nucleus is commonly present in a large number of natural products as well as pharmacologically active compounds [1]. It shows a wide spectrum of biological and pharmacological properties such as antifungal [2], antimicrobial [3], anthelmintic $[4,5]$, antiviral $[6,7]$, topoisomerase inhibition [8] and anticancer activities [9]. Some of their derivatives are marketed as antifungal drug (Carbendazim) [10], anthelmintic drug (Mebendazole and Thiabendazole) [11], antipsychotic drug (Pimozide) [12] and antiulcer agent (Omeprazole) [13]. Owing to their interesting pharmacological properties, great attention has been paid to the synthesis of benzimidazoles. Two main synthetic methods were well known in the literature. The most common method is direct condensation of 1,2-phenylenediamine and carboxylic acids $[14,15]$ or their derivatives [16], that require strong acidic conditions and sometimes need high temperature or the use of microwave [17]. The other synthetic route involves a two-step procedure that includes the cyclo-dehydrogenation of aniline Schiff's bases, which are often generated in situ from the condensation of 1,2-phenylenediamines and aldehydes [18], followed by oxidation with stoichiometric amount of oxidants, such as $\mathrm{MnO}_{2}$ [19], Oxone [20], $\mathrm{NaHSO}_{3}[21,22]$, $\mathrm{I}_{2} / \mathrm{KI} / \mathrm{K}_{2} \mathrm{CO}_{3} / \mathrm{H}_{2} \mathrm{O}$ [23] or catalytic use of CAN [24] and AIKIT-5 [25]. More recently, 2-alkyl substituted benzimidazoles are synthesized by using hexafluorophosphoric acid under microwave condition [26].

There is renewed interest in the silica supported catalyzed reactions [27]. These reactions have relatively shorter reaction time with high yield and cleaner chemistry. Moreover, the catalyst is easily separated from reaction mixture by simple filtration. There are very few reports involving solid supported catalyzed reaction for synthesis of benzimidazole derivatives. Jacob et al. [28] synthesized 1,2-disubstituted benzimidazoles by silica supported $\mathrm{ZnCl}_{2}$ catalyst that was found to be of poor yield. Patil et al. [29] developed a method for synthesis of 2-alkyl benzimidazoles using silica supported $\mathrm{HBF}_{4}$. Paul and Basu [30] described the synthesis of 1,2-disubstituted benzimidazoles by using silica gel soaked with $\mathrm{Fe}_{2}\left(\mathrm{SO}_{4}\right)_{3}$. $x \mathrm{H}_{2} \mathrm{O}$. Recently, Kumar et al. [31] reported silica supported $\mathrm{HClO}_{4}$ catalyzed synthesis of benzimidazoles.

Periodic acid is an easily available hypervalent iodine reagent which is used in the oxidation of various functional groups $[32,33]$. However, there are no reported efforts for the synthesis of benzimidazoles by using periodic acid. In 
this paper, we report an efficient and facile synthesis of 2aryl benzimidazoles by using silica supported periodic acid $\left(\mathrm{H}_{5} \mathrm{IO}_{6}-\mathrm{SiO}_{2}\right)$ as a catalyst (Scheme 1$)$. Further, all synthesized derivatives were screened for anticancer activity against two cancer cell lines, namely, MCF7 and HL60.

\section{Result and Discussion}

Herein, we used unprecedented silica supported periodic acid $\left(\mathrm{H}_{5} \mathrm{IO}_{6}-\mathrm{SiO}_{2}\right)$ as catalyst for synthesis of 2-aryl benzimidazole derivatives. In our initial experiments, we choose 1,2-phenylenediamine $(1 \mathrm{mmol})$ and $m$-nitrobenzaldehyde $(1 \mathrm{mmol})$ as a model reaction for optimization of catalyst and reaction conditions. The results are summarized in Table 1. The use of $20 \mathrm{~mol} \%$ of $\mathrm{H}_{5} \mathrm{IO}_{6}$ catalyst supported on silica resulted in $95 \%$ of desired product, $5 \mathrm{~g}$ in $15 \mathrm{~min}$ at room temperature (Table 1, entry 1). Inferior yields were obtained on lowering the catalyst loading at room temperature (Table 1, entries 2 and 3). Even, on increasing temperature, the yield was not improved (Table 1 , entries 6 to 8 ). In control experiments, the poor yields were found in the absence of $\mathrm{H}_{5} \mathrm{IO}_{6}$ (Table 1, entries 5 and 10) at room temperature and $60^{\circ} \mathrm{C}$ even after prolonged time $(10 \mathrm{~h})$. Further, the reactions were carried out with only $\mathrm{H}_{5} \mathrm{IO}_{6}$ without silica support at room temperature and $60^{\circ} \mathrm{C}$ (Table 1 , entries 4 and 9) where the yield was not found to be more than $35 \%$. These results confirmed that $\mathrm{H}_{5} \mathrm{IO}_{6}$ supported on silica significantly increased the efficacy of catalysts which may be attributed to the increase in available surface area. Thus, we found optimized conditions as the 1,2-phenylenediamine ( $1 \mathrm{mmol})$, aldehyde $(1 \mathrm{mmol})$ and $\mathrm{H}_{5} \mathrm{IO}_{6}(0.20 \mathrm{mmol}$ supported on silica) in acetonitrile ( $\mathrm{ACN})$ at room temperature.

Feasibility of the methodology was examined for a series of aryl/heteroaryl aldehydes bearing electron donating as well as electron withdrawing groups under the optimized reaction conditions and corresponding products were obtained in good to excellent yields (Table 2). Presence of electron withdrawing group in aldehyde system fastened the reaction (entries 5f-5h) while opposite effect was observed for electron donating substituents and hindered aldehydes (entries $\mathbf{5 b}$ and $\mathbf{5 h}$ ). We have not observed any remarkable change in the reaction time for the different substituted diamines. The reaction underwent smoothly even with aldehyde bearing two functional groups (entries 5c, 5h, 6c \& 7c) and afforded corresponding products in good yields (entry $5 \mathbf{h}$ ). The reaction was carried out with substituted 1,2-phenylenediamine (entries 6a-6e and 7a-7c) and afforded 2,5-substituted benzimidazoles in moderate to good yields. Using these reaction conditions exclusively formation of 2-substituted benzimidazoles was observed. The products were characterized by their physical and spectral data. Thus, Table 2 illustrates generality and efficiency of this method for the synthesis of benzimidazoles.

We have also extended same methodology for the synthesis of bisbenzimidazole (8) derivative by using 1,2-phenylenediamine, aryl aldehyde $(2: 1)$ affording compound $\mathbf{8}$ in $80 \%$ yield (Scheme 2).

Although the exact mechanism is not clear, a proposed mechanism for the formation of benzimidazole is shown in
TABLE 1: Reaction of $\mathbf{1}$ and $\mathbf{4} \mathbf{g}$ under various conditions ${ }^{\mathrm{a}}$.

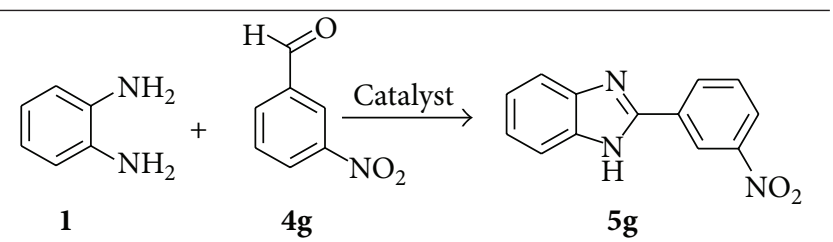

\begin{tabular}{lcccc}
\hline Entry & $\begin{array}{c}\text { Catalyst }(\mathrm{mol} \%) \\
\text { on silica }\end{array}$ & Temp. $^{\mathrm{b}}$ & Time & Yield $(\%)^{\mathrm{c}}$ \\
\hline $\mathbf{1}$ & $\mathrm{H}_{5} \mathrm{IO}_{6}(20)$ & $\mathrm{rt}$ & $15 \mathrm{~min}$ & 95 \\
$\mathbf{2}$ & $\mathrm{H}_{5} \mathrm{IO}_{6}(15)$ & $\mathrm{rt}$ & $15 \mathrm{~min}$ & 70 \\
$\mathbf{3}$ & $\mathrm{H}_{5} \mathrm{IO}_{6}(10)$ & $\mathrm{rt}$ & $15 \mathrm{~min}$ & 45 \\
$\mathbf{4}$ & $\mathrm{H}_{5} \mathrm{IO}_{6}(20)^{\mathrm{d}}$ & $\mathrm{rt}$ & $10 \mathrm{~h}$ & 28 \\
$\mathbf{5}$ & $\mathrm{SiO}_{2}$ & $\mathrm{rt}$ & $10 \mathrm{~h}$ & 12 \\
$\mathbf{6}$ & $\mathrm{H}_{5} \mathrm{IO}_{6}(20)$ & $60^{\circ} \mathrm{C}$ & $15 \mathrm{~min}$ & 94 \\
$\mathbf{7}$ & $\mathrm{H}_{5} \mathrm{IO}_{6}(15)$ & $60^{\circ} \mathrm{C}$ & $15 \mathrm{~min}$ & 67 \\
$\mathbf{8}$ & $\mathrm{H}_{5} \mathrm{IO}_{6}(10)$ & $60^{\circ} \mathrm{C}$ & $15 \mathrm{~min}$ & 47 \\
$\mathbf{9}$ & $\mathrm{H}_{5} \mathrm{IO}_{6}(20)^{\mathrm{d}}$ & $60^{\circ} \mathrm{C}$ & $10 \mathrm{~h}$ & 35 \\
$\mathbf{1 0}$ & $\mathrm{SiO}_{2}$ & $60^{\circ} \mathrm{C}$ & $10 \mathrm{~h}$ & 16 \\
\hline
\end{tabular}

${ }^{\mathrm{a}} 1,2$-phenylenediamine $1 \mathrm{mmol}, m$-nitrobenzaldehyde $1 \mathrm{mmol}$ catalyst ( 0.20 mmol supported on silica) in ACN.

${ }^{\mathrm{b}}$ Room temperature was $30-35^{\circ} \mathrm{C}$.

${ }^{c}$ Yields are measured after purification.

${ }^{\mathrm{d}} \mathrm{H}_{5} \mathrm{IO}_{6}$ which is not supported on silica.

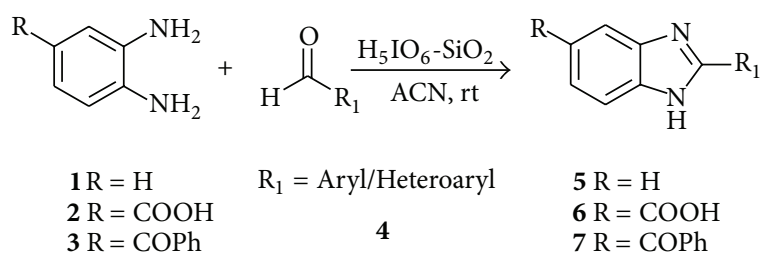

Scheme 1: Synthesis of 2-aryl benzimidazole.

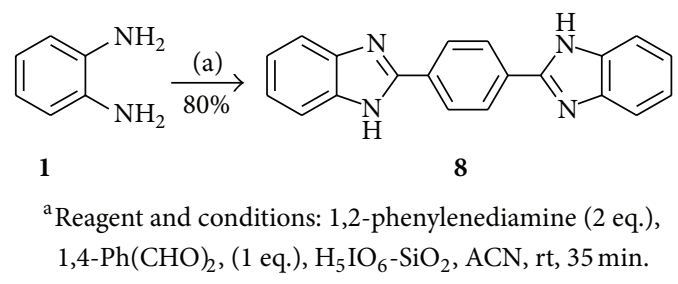

Scheme 2: Synthesis of bisbenzimidazole.

Scheme 3. The actual oxidant is $\mathrm{H}_{5} \mathrm{IO}_{6}-\mathrm{SiO}_{2}$ and not $\mathrm{SiO}_{2}$ as confirmed by our controlled experiments (Table 1). The acidic site of catalyst is anticipated for oxidation [34].

\section{Anticancer Activity}

All the synthesized benzimidazoles were tested for their anticancer activity against two cell lines MCF7 (human breast adenocarcinoma) and HL60 (human promyelocytic leukemia) by MTT colorimetric assay using cisplatin as a standard anticancer drug. The results are expressed as $\mathrm{IC}_{50}$ in $\mu \mathrm{M}$ 


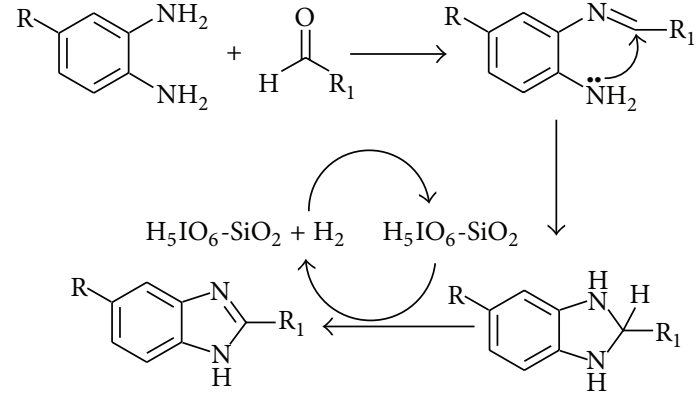

Scheme 3: Plausible mechanism towards the formation of 2-aryl benzimidazole.

and summarized in Table 2. Anticancer activity varies with substitution at 5-position of benzimidazole ring. The benzoyl substituted benzimidazole $(\mathbf{7 a}-7 \mathbf{c})$ showed the highest potency against two cell lines, while carboxyl substituted compounds (6a and $\mathbf{6 b}$ ) were moderately potent (with the exception of $6 \mathrm{c}$ ), as compared to unsubstituted benzimidazole $(\mathbf{5 a}-\mathbf{5} \mathbf{c})$. Dichloro derivatives $(\mathbf{5} \mathbf{c}$ and $\mathbf{6 c})$ exhibited more activity as compared to monochloro derivatives (5d and $\mathbf{6 d}$ ) against MCF7 and HL60. Substitution of nitro group (5e5 g) showed moderate and mostly similar effect for the given cell lines. Compound $\mathbf{5 b}$ with phenolic $-\mathrm{OH}$ group $\left(\mathrm{IC}_{50}\right.$ $27.63 \mu \mathrm{M}$ for MCF7 and 28.68 $\mu \mathrm{M}$ for HL60) was comparable to $5 \mathbf{h}\left(\mathrm{IC}_{50} 29.67 \mu \mathrm{M}\right.$ for MCF7 and $26.52 \mu \mathrm{M}$ for HL60) which has additional methoxy group at $p$-position. Replacing ring carbon of benzene ring with nitrogen atom as in $\mathbf{5 i}$ $\left(\mathrm{IC}_{50} 30.42 \mu \mathrm{M}\right)$ showed better activity against MCF7 when compared with $5 \mathbf{a}\left(\mathrm{IC}_{50} 35.67 \mu \mathrm{M}\right)$, but same compound did not show substantial difference in activity against HL60. Bisbenzimidazole (8) was found to be more active for MCF7 $\left(\mathrm{IC}_{50} 17.45 \mu \mathrm{M}\right)$ than HL60 $\left(\mathrm{IC}_{50} 30.69 \mu \mathrm{M}\right)$. All the tested compounds are found to be more effective against both cell lines as compared to cisplatin.

\section{Conclusion}

We have developed a short and efficient method for the synthesis of 2-aryl benzimidazoles from 1,2-phenylenediamines and aryl aldehydes using $\mathrm{H}_{5} \mathrm{IO}_{6}-\mathrm{SiO}_{2}$ as catalyst. The mild reaction condition, low cost, easy workup procedure and good to excellent yields as well as the scope for using wide substrates make our methodology a valuable contribution to the existing processes for synthesis of benzimidazole derivatives. Among 18 derivatives, newly synthesized 5-substituted derivatives exhibited excellent activity against MCF7 and HL60 cell lines. The overall activities for all the derivatives tested were found in micromolar range. The current study provides better insight into the designing of more potent anticancer agents in the future.

\section{Experimental Section}

All reactions were performed in open atmosphere with unpurified reagents and distilled solvents. Periodic acid was purchased from Spectrochem. Acetonitrile and silica (230-400) were purchased from Sigma Aldrich. Thin-layer chromatography (TLC) was performed using $0.25 \mathrm{~mm}$ silica gel coated plates. Column chromatography was performed using the hexane: ethylacetate solvent and silica gel (60-120 meshes). ${ }^{1} \mathrm{H}$ NMR $(300 \mathrm{MHz})$ and ${ }^{13} \mathrm{CNMR}(75 \mathrm{MHz})$ spectra were recorded on Varian Mercury instrument with DMSO- $d_{6}$ or $\mathrm{D}_{2} \mathrm{O}$ as the solvents. Chemical shifts were reported in $\delta$ unit (ppm) with reference to TMS as an internal standard, and $J$ values were given in Hertz. Melting points were determined on Thomas Hoover capillary melting point apparatus and are uncorrected. IR spectra were recorded on a Shimadzu FTIR 8400 spectrophotometer in $\mathrm{KBr}$ disc and expressed in $\mathrm{cm}^{-1}$. Elemental analysis was carried out with Thermo-Electron Corporation CHNS analyzer Flash-EA 1112.

5.1. Cell Culture. Two cancer cell lines, MCF7 (human breast adenocarcinoma) and HL60 (human promyelocytic leukemia), were obtained from National Center for Cell Sciences, India. MCF7 was cultured in DMEM medium [35] while HL60 cells were cultured in a humidified atmosphere $\left(37^{\circ} \mathrm{C}\right.$, $5 \% \mathrm{CO}_{2}$ ) in RPMI1640 medium supplemented with $10 \%$ fetal bovine serum.

5.2. MTT Assay. Test compounds were evaluated for anticancer activity against two cancer cell lines using cisplatin as standard anticancer drug. The compounds were evaluated in vitro at a concentration range of $10 \mu \mathrm{M}$ to $100 \mu \mathrm{M}$. The MTT colorimetric assay was used to determine growth inhibition. $100 \mu \mathrm{L}$ of cell suspension $\left(5 \times 10^{6}\right.$ cells $)$ were plated in $96-$ well plates and allowed to attach for $24 \mathrm{~h}$. The compounds were dissolved in $0.5 \%$ DMSO. Cells were exposed in triplicate wells to these derivatives at various concentrations for $48 \mathrm{~h}$. After $48 \mathrm{~h}, 20 \mu \mathrm{L}$ MTT (3-(4,5-dimethylthiazol-2-yl)2,5-diphenyltetrazolium bromide) solution $(5 \mathrm{mg} / \mathrm{mL})$ was added to each well. After $1 \mathrm{~h}$ of incubation, the solution was centrifuged for $5 \mathrm{~min}$ under $4000 \mathrm{rpm}$, and the medium was discarded carefully. The formazan precipitate was dissolved in DMSO $(200 \mu \mathrm{L})$, then shaken by oscillator. The absorbance at $570 \mathrm{~nm}$ was determined on a microplate reader (Bio-Rad Model 3350, Japan). The absorbance values were used to calculate $\%$ inhibition at various concentrations and $\mathrm{IC}_{50}$ values.

5.3. Procedure for Synthesis of Silica Supported $\mathrm{H}_{5} \mathrm{IO}_{6}$ Catalyst $\left(\mathrm{H}_{5} \mathrm{IO}_{6}-\mathrm{SiO}_{2}\right) . \mathrm{H}_{5} \mathrm{IO}_{6}(2.50 \mathrm{~g}, 10.96 \mathrm{mmol})$ was dissolved in $15 \mathrm{~mL}$ of hot water $\left(70^{\circ} \mathrm{C}\right)$ in a $50 \mathrm{~mL}$ round-bottomed flask. To the hot solution was added silica gel (230-400 meshes, $10 \mathrm{~g}$ ) with vigorous stirring. The resultant $\mathrm{H}_{5} \mathrm{IO}_{6}$ (resultant mixture contains $20 \mathrm{wt} \%$ of $\mathrm{H}_{5} \mathrm{IO}_{6}$ ) supported with silica gel was dried in oven at $100^{\circ} \mathrm{C}$ for $12 \mathrm{~h}$ to obtain a white free flow powder. The reagent can be stored for 4 months with negligible loss of activity.

5.4. General Procedure for Synthesis of Benzimidazoles (5a-5i, $\mathbf{6 a}-\mathbf{6} \boldsymbol{e}$ and $7 \boldsymbol{a}-7 \mathrm{c})$. A typical procedure is as follows. A mixture of 1,2-phenylenediamine (108 mg, $1.0 \mathrm{mmol}$ ), $m$-nitrobenzaldehyde $(151 \mathrm{mg}, 1.0 \mathrm{mmol})$ in acetonitrile $(3.0 \mathrm{~mL})$ was taken, and $\mathrm{H}_{5} \mathrm{IO}_{6}(45 \mathrm{mg}, 20 \mathrm{mmol} \%$ supported on silica $210 \mathrm{mg}$ ) was added at room temperature. The reaction was stirred at room temperature for 15 minutes. After completion 
TABLE 2: Synthesis and anticancer activity of 2-aryl benzimidazoles against MCF7 and HL60 cell lines.

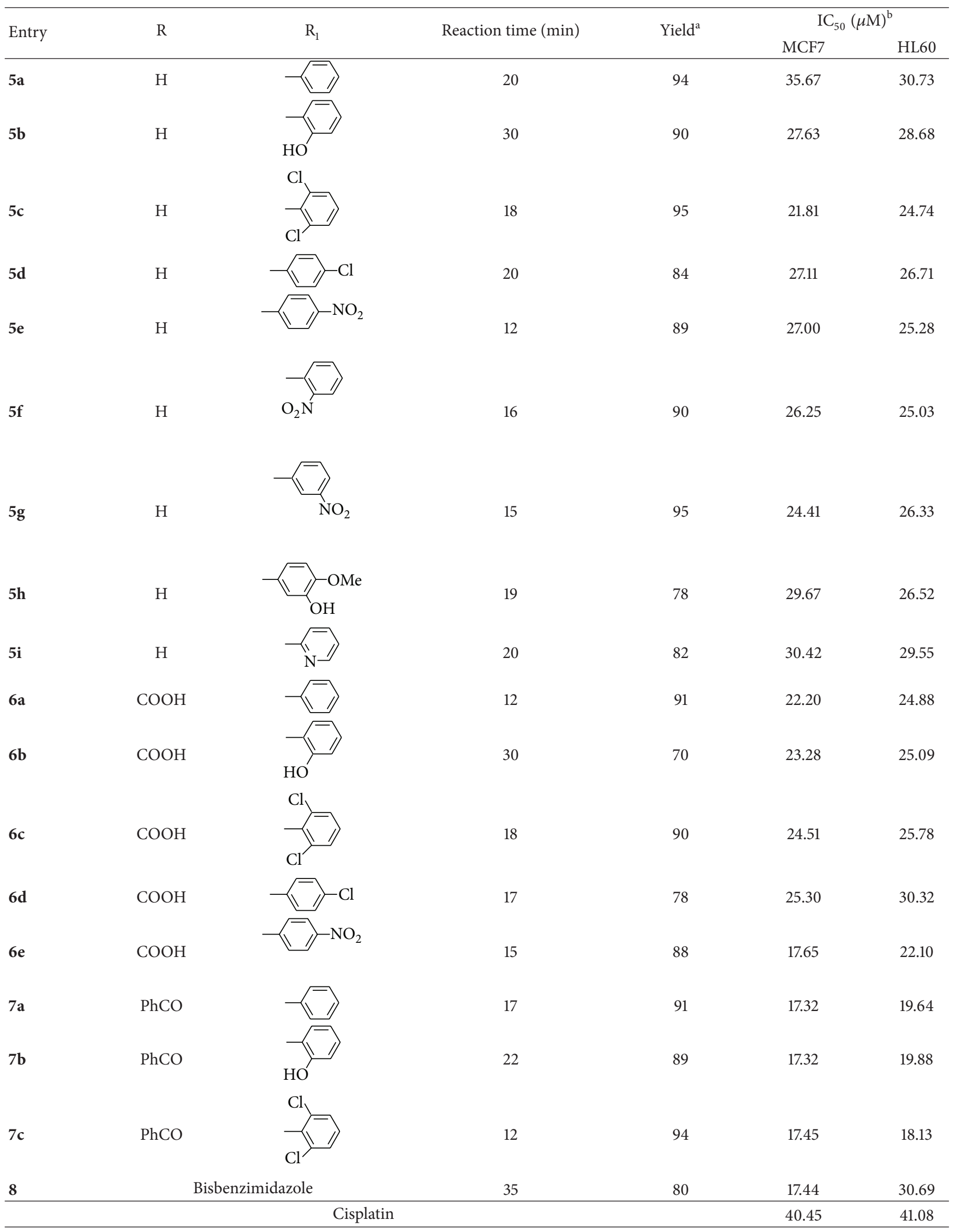

${ }^{\mathrm{a}} 1,2$-phenylenediamine $1 \mathrm{mmol}, \mathrm{m}$-nitrobenzaldehyde $1 \mathrm{mmol}$ catalyst $(0.20 \mathrm{mmol}$ supported on silica) in ACN. Isolated yields after purification.

${ }^{\mathrm{b}} \mathrm{IC}_{50} 50 \%$ inhibition concentration in $\mu \mathrm{M}$. 
of the reaction (monitored by TLC), filter the reaction mixture over celite. The filtrate was evaporated under vacuum and subsequently dried to afford crude product which was purified by column chromatography using hexane/ethylacetate as eluent to afford pure benzimidazole $5 \mathrm{~g}$ (227 $\mathrm{mg}$, 95\%).

5.5. Procedure for Synthesis of Bisbenzimidazole (8). A mixture of 1,2-phenylenediamine (216 mg, $2.0 \mathrm{mmol})$, p-phthalaldehyde $(134 \mathrm{mg}, 1.0 \mathrm{mmol})$ in acetonitrile $(3.0 \mathrm{~mL})$ was taken, and $\mathrm{H}_{5} \mathrm{IO}_{6}$ (90 mg, $40 \mathrm{~mol} \%$ supported on silica $420 \mathrm{mg}$ ) was added at room temperature. The reaction was stirred at room temperature for 35 minutes. After completion of the reaction (monitored by TLC), the reaction mixture was filtered over celite. The filtrate was evaporated under vacuum and subsequently dried to afford crude product which was purified by column chromatography using hexane/ethylacetate as eluent to afford pure benzimidazole $\mathbf{8}(250 \mathrm{mg}, 80 \%)$. The spectral data are in full agreement with data reported in the literature. Spectral data of compounds are given below.

5.5.1. 2-Phenyl-1H-benzoimidazole (5a). White solid; mp 291-293 ${ }^{\circ}$; (lit. [21, 22] mp 290-291 ${ }^{\circ} \mathrm{C}$ ); IR ( $\left.\mathrm{cm}^{-1}, \mathrm{KBr}\right)$ : $3044,1622,1587,1537,1458,1439,1407,1312,1274 ;{ }^{1} \mathrm{H}$ NMR $\left(300 \mathrm{MHz}, \mathrm{DMSO}-d_{6}\right): \delta 12.91$ (brs, $\left.1 \mathrm{H}\right), 8.15(\mathrm{~d}, J=7.0 \mathrm{~Hz}$, 2H), 7.55-7.47 (m, 5H), 7.19 (brs, $2 \mathrm{H}) ;{ }^{13} \mathrm{C}$ NMR $(75 \mathrm{MHz}$, DMSO- $\left.d_{6}\right): \delta 151.2,143.7,134.9,130.1,129.8,128.9,126.4,122.4$, 121.6, 118.8, 111.3; (Found: C, 80.39; H, 5.18; N, 14.38. Cal for $\mathrm{C}_{13} \mathrm{H}_{10} \mathrm{~N}_{2}$ : C, 80.42; H, 5.19; N, 14.42\%).

5.5.2. 2-(1H-Benzo[d]imidazol-2-yl) Phenol (5b). White solid; $\mathrm{mp} 235-237^{\circ} \mathrm{C}$; (lit. [21, 22] mp 236-237 $\left.{ }^{\circ} \mathrm{C}\right)$; IR $\left(\mathrm{cm}^{-1}\right.$, $\mathrm{KBr}): 3327,3057,2332,1635,1280,1037,840,729 ;{ }^{1} \mathrm{H}$ NMR $\left(300 \mathrm{MHz}, \mathrm{DMSO}-d_{6}\right.$ ): $\delta 13.21$ (brs, $\left.2 \mathrm{H}\right), 8.07$ (d, $J=7.7 \mathrm{~Hz}$, 1H), 7.67 (brs, 2H), 7.28-7.40 (m, 3H), 6.99-7.06 (m, 2H); ${ }^{13} \mathrm{C}$ NMR $\left(75 \mathrm{MHz}, \mathrm{DMSO}-d_{6}\right): \delta 158.0,151.7,131.6,126.2,122.7$, 119.0, 117.1, 112.5; (Found: C, 74.25; H, 4.78; N, 13.31. Cal for $\left.\mathrm{C}_{13} \mathrm{H}_{10} \mathrm{~N}_{2} \mathrm{O}: \mathrm{C}, 74.27 ; \mathrm{H}, 4.79 ; \mathrm{N}, 13.33 \%\right)$.

5.5.3. 2-(2,6-Dichlorophenyl)-1H-benzimidazole (5c). White solid; mp 274-276 ${ }^{\circ}$; (lit. [36] mp 275-276 $\left.{ }^{\circ} \mathrm{C}\right)$; IR $\left(\mathrm{cm}^{-1}\right.$, $\mathrm{KBr}): 3368,3297,1558,1431,1369,1265,1132,735 ;{ }^{1} \mathrm{H}$ NMR $\left(300 \mathrm{MHz}, \mathrm{DMSO}-d_{6}\right): \delta 12.90$ (brs, $\left.1 \mathrm{H}\right), 7.53-7.71(\mathrm{~m}, 5 \mathrm{H})$, 7.20-7.29 (m, 2H); ${ }^{13} \mathrm{C}$ NMR (75 MHz, DMSO- $\left.d_{6}\right): \delta$ 146.7, 143.1, 135.0, 134.0, 132.3, 130.5, 128.3, 122.8, 121.6, 119.2, 111.6; (Found: $\mathrm{C}, 59.33 ; \mathrm{H}, 3.05 ; \mathrm{N}, 10.62$. Cal for $\mathrm{C}_{13} \mathrm{H}_{8} \mathrm{Cl}_{2} \mathrm{~N}_{2}$ : C, $59.34 ; \mathrm{H}, 3.06 ; \mathrm{N}, 10.65 \%)$.

5.5.4. 2-(4-Chlorophenyl)-1H-benzimidazole (5d). White solid; mp 288-291 ${ }^{\circ} \mathrm{C}$; (lit. [25] 287-289 $\left.{ }^{\circ} \mathrm{C}\right)$; IR ( $\left.\mathrm{cm}^{-1}, \mathrm{KBr}\right) 3433$, 3055, 1427, 1273, 1091, 829, 744; ${ }^{1} \mathrm{H}$ NMR (300 MHz, DMSO$d_{6}$ ): $\delta 13.00$ (brs, $\left.1 \mathrm{H}\right), 8.23(\mathrm{~d}, J=8.2 \mathrm{~Hz}, 2 \mathrm{H}), 7.63(\mathrm{~d}, J=$ $8.5 \mathrm{~Hz}, 2 \mathrm{H}), 7.54(\mathrm{~d}, J=5.9 \mathrm{~Hz}, 2 \mathrm{H}), 7.21$ (brs, $2 \mathrm{H}) ;{ }^{13} \mathrm{C} \mathrm{NMR}$ $\left(75 \mathrm{MHz}, \mathrm{DMSO}-d_{6}\right): \delta 150.2,143.7,134.5,129.1,129.0,128.1$, 122.4, 118.9, 111.5; (Found: C, 68.27; H, 3.95; N, 12.21. Cal for $\mathrm{C}_{13} \mathrm{H}_{9} \mathrm{ClN}_{2}$ : C, 68.28; H, 3.97; N, 12.25\%).

5.5.5. 2-(4-Nitrophenyl)-1H-benzo[d]imidazole (5e). Yellow solid; mp 300-302 $\mathrm{C}$; (lit. [21, 22] mp 299-301 $\left.{ }^{\circ} \mathrm{C}\right)$; IR $\left(\mathrm{cm}^{-1}\right.$, $\mathrm{KBr})$ : 3335, 2912, 1602, 1514, 1435, 1340, 1103, 856, 746;
${ }^{1} \mathrm{H}$ NMR (300 MHz, DMSO- $\left.d_{6}\right): \delta 13.39$ (brs, $\left.1 \mathrm{H}\right), 8.38-8.27$ (m, 4H), 7.61 (s, 2H), 7.22-7.20 (m, 2H); ${ }^{13} \mathrm{C}$ NMR (75 MHz, DMSO- $\left.d_{6}\right): \delta 148.8,147.5,142.7,135.9,127.2,124.1,124.0,122.8$; (Found: C, 65.25; $\mathrm{H}, 3.75 ; \mathrm{N}, 17.52$. Cal for $\mathrm{C}_{13} \mathrm{H}_{9} \mathrm{~N}_{3} \mathrm{O}_{2}$ : C, $65.27 ; \mathrm{H}, 3.79 ; \mathrm{N}, 17.56 \%)$.

5.5.6. 2-(2-Nitrophenyl)-1H-benzo[d]imidazole (5f). Yellow solid; mp 209-211 ${ }^{\circ} \mathrm{C}$; (lit. [25] 208-210 $\left.{ }^{\circ} \mathrm{C}\right)$; IR (cm $\left.{ }^{-1}, \mathrm{KBr}\right)$ : 3410, 3078, 2686, 1525, 1348, 1078, 972, 746; ${ }^{1} \mathrm{H}$ NMR (300 MHz, DMSO- $\left.d_{6}\right): \delta 13.07$ (brs, $\left.1 \mathrm{H}\right), 7.96-8.04(\mathrm{~m}, 2 \mathrm{H})$, 7.83-7.89 (m, 1H), 7.72-7.78 (m, 1H), 7.60-7.63 (m, 2H), 7.23$7.26(\mathrm{~m}, 2 \mathrm{H}) ;{ }^{13} \mathrm{C}$ NMR $\left(75 \mathrm{MHz}, \mathrm{DMSO}-d_{6}\right): \delta 148.9,147.2$, 132.5, 131.4, 130.8, 130.8, 128.6, 124.2, 122.4, 119.1, 111.5; (Found: C, 65.26; $\mathrm{H}, 3.74 ; \mathrm{N}, 17.53$. Cal for $\mathrm{C}_{13} \mathrm{H}_{9} \mathrm{~N}_{3} \mathrm{O}_{2}$ : C, 65.27; $\mathrm{H}$, $3.79 ; \mathrm{N}, 17.56 \%)$.

5.5.7. 2-(3-Nitrophenyl)-1H-benzimidazole (5g). Yellow solid; $\mathrm{mp} 205-207^{\circ} \mathrm{C}$; (lit. [21, 22] mp 205-206 C); IR $\left(\mathrm{cm}^{-1}\right.$, $\mathrm{KBr}): 3371,3088,1587,1518,1473,1429,1346,1274 ;{ }^{1} \mathrm{H}$ NMR $\left(300 \mathrm{MHz}, \mathrm{DMSO}-d_{6}\right) \delta 13.29(\mathrm{~s}, 1 \mathrm{H}), 9.01(\mathrm{~s}, 1 \mathrm{H}), 8.60(\mathrm{~d}, J=$ $7.6 \mathrm{~Hz}, 1 \mathrm{H}), 8.32(\mathrm{~d}, J=7.9 \mathrm{~Hz}, 1 \mathrm{H}), 7.84(\mathrm{t}, J=7.9 \mathrm{~Hz}, 1 \mathrm{H})$, $7.72(\mathrm{~d}, J=7.3 \mathrm{~Hz}, 1 \mathrm{H}), 7.58(\mathrm{~d}, J=7.4 \mathrm{~Hz}, 1 \mathrm{H}), 7.25(\mathrm{t}, J=$ $6.7 \mathrm{~Hz}, 2 \mathrm{H}) ;{ }^{13} \mathrm{C}$ NMR $\left(75 \mathrm{MHz}, \mathrm{DMSO}-d_{6}\right): \delta 148.9,148.2$, $143.5,134.9,132.3,131.6,130.5,124.0,123.1,122.0,120.7,119.1$, 111.5; (Found: C, 66.26; H, 3.75, N; 17.55. Cal for $\mathrm{C}_{13} \mathrm{H}_{9} \mathrm{~N}_{3} \mathrm{O}_{2}$ : C, 65.27; H, 3.79; N, 17.56\%).

5.5.8. 5-(1H-Benzo[d]imidazol-2-yl)-2-methoxyphenol (5h). Yellow solid; mp: $218-220^{\circ} \mathrm{C}$; IR $\left(\mathrm{cm}^{-1}, \mathrm{KBr}\right): 3273,2926$, $1500,1450,1265,1033,910,736 ;{ }^{1} \mathrm{H}$ NMR (300 MHz, DMSO$\left.d_{6}\right): \delta 12.65(\mathrm{~s}, 1 \mathrm{H}), 9.31(\mathrm{~s}, 1 \mathrm{H}), 7.52-7.63(\mathrm{~m}, 4 \mathrm{H}), 7.13-7.19(\mathrm{~m}$, $2 \mathrm{H}), 7.05(\mathrm{~d}, J=8.2 \mathrm{~Hz}, 1 \mathrm{H}), 3.85(\mathrm{~s}, 3 \mathrm{H}) ;{ }^{13} \mathrm{C} \mathrm{NMR}(75 \mathrm{MHz}$, DMSO- $\left.d_{6}\right): \delta 151.5,149.3,146.6,139.9,122.8,121.6,117.9,114.4$, 113.7, 112.0, 55.6; (Found: C, 69.91; H, 4.99; N, 11.65. Cal for $\mathrm{C}_{14} \mathrm{H}_{12} \mathrm{~N}_{2} \mathrm{O}_{2}$ : C, 69.99; $\left.\mathrm{H}, 5.03 ; \mathrm{N}, 11.66 \%\right)$.

5.5.9. 2-(Pyridine-2-yl)-1H-benzo[d)imidazole (5i). Yellow solid; mp 218-220 ${ }^{\circ}$; IR ( $\left.\mathrm{cm}^{-1}, \mathrm{KBr}\right): 3057,2667,1595,1444$, $1315,1280,1122,850,744,615 ;{ }^{1} \mathrm{H}$ NMR (300 MHz, DMSO$\left.d_{6}\right): \delta 13.11$ (brs, $\left.1 \mathrm{H}\right), 8.72(\mathrm{~d}, J=4.8 \mathrm{~Hz}, 1 \mathrm{H}), 8.33(\mathrm{~d}, J=$ $8.1 \mathrm{~Hz}, 1 \mathrm{H}), 8.02(\mathrm{t}, J=7.7 \mathrm{~Hz}, 1 \mathrm{H}), 7.49-7.69$ (m, 3H), 7.21-7.24 $(\mathrm{m}, 2 \mathrm{H}) ;{ }^{13} \mathrm{C}$ NMR $\left(75 \mathrm{MHz}, \mathrm{DMSO}-d_{6}\right): \delta 151.2,150.1,148.3$, 138.3, 125.6, 123.6, 122.0, 116.2; (Found: C, 78.80; H, 4.60; N, 21.51. Cal for $\mathrm{C}_{12} \mathrm{H}_{9} \mathrm{~N}_{3}$ : C, 78.83; $\mathrm{H}, 4.65 ; \mathrm{N}, 21.52 \%$ ).

5.5.10. 2-Phenyl-1H-benzo[d]imidazole-5-carboxylic Acid

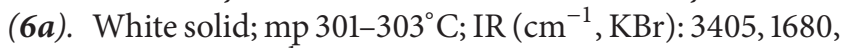
1621, 1450, 981, 770; ${ }^{1} \mathrm{H}$ NMR (300 MHz, DMSO- $d_{6}$ ): $\delta 13.18$ (brs, $1 \mathrm{H}), 12.79$ (brs, $1 \mathrm{H}), 8.19(\mathrm{~m}, 3 \mathrm{H}), 7.84$ (d, $J=8.5 \mathrm{~Hz}$, $1 \mathrm{H}), 7.63(\mathrm{~d}, J=15.2 \mathrm{~Hz}, 1 \mathrm{H}), 7.58-7.48(\mathrm{~m}, 3 \mathrm{H}) ;{ }^{13} \mathrm{C} \mathrm{NMR}$ $\left(75 \mathrm{MHz}, \mathrm{DMSO}-d_{6}\right): \delta 193.2,167.8,153.5,134.5,130.4,129.5$, 129.2, 129.0, 128.5, 126.7, 124.6, 123.6, 117.1, 114.6; (Found: C, 70.55; $\mathrm{H}, 4.24 ; \mathrm{N}, 11.72$. Cal for $\mathrm{C}_{14} \mathrm{H}_{10} \mathrm{~N}_{2} \mathrm{O}_{2}$ : C, 70.58; $\mathrm{H}$, $4.23 ; \mathrm{N}, 11.76 \%)$.

5.5.11. 2-(2-Hydroxyphenyl)-1H-benzo[d]imidazole-5-carboxylic Acid (6b). White solid; mp $301-303^{\circ} \mathrm{C}$; IR $\left(\mathrm{cm}^{-1}, \mathrm{KBr}\right)$ : 3319, 3059, 1681, 1633, 1491, 1261, 1130, 748; ${ }^{1} \mathrm{H}$ NMR 
(300 MHz, DMSO- $d_{6}$ ): $\delta 13.43$ (brs, $\left.1 \mathrm{H}\right), 12.84$ (brs, $\left.2 \mathrm{H}\right), 8.27$ (brs, $1 \mathrm{H}), 8.10$ (d, $J=7.2 \mathrm{~Hz}, 1 \mathrm{H}), 7.90$ (brs, $1 \mathrm{H}), 7.72$ (brs, $1 \mathrm{H})$, $7.43(\mathrm{t}, J=7.4 \mathrm{~Hz}, 1 \mathrm{H}), 7.07(\mathrm{~d}, J=8.6 \mathrm{~Hz}, 2 \mathrm{H}) ;{ }^{13} \mathrm{C} \mathrm{NMR}$ $\left(75 \mathrm{MHz}, \mathrm{DMSO}-d_{6}\right): \delta 168.3,158.0,153.9,132.9,132.0,129.1$, 127.3, 125.5, 124.6, 120.1, 117.6, 112.8; (Found: C, 66.16; H, 3.93; $\mathrm{N}, 10.09$. Cal for $\mathrm{C}_{14} \mathrm{H}_{10} \mathrm{~N}_{2} \mathrm{O}_{3}$ : C, 66.14; $\left.\mathrm{H}, 3.96 ; \mathrm{N}, 11.02 \%\right)$.

5.5.12. 2-(2,6-Dichlorophenyl)-1H-benzo[d]imidazole-5-carboxylic Acid (6c). White solid; mp 304-306 ${ }^{\circ}$; IR $\left(\mathrm{cm}^{-1}\right.$, $\mathrm{KBr}): 3171,1915,1668,1622,1433,1315,779 ;{ }^{1} \mathrm{H}$ NMR $\left(300 \mathrm{MHz}, \mathrm{DMSO}-d_{6}\right)$ : 13.12 (brs, $\left.1 \mathrm{H}\right), 12.64$ (brs, $\left.1 \mathrm{H}\right)$, 8.28-8.17 (m, 1H), 7.88 (d, $J=7.6 \mathrm{~Hz}, 1 \mathrm{H}), 7.72-7.57(\mathrm{~m}, 4 \mathrm{H})$; ${ }^{13} \mathrm{C}$ NMR $\left(75 \mathrm{MHz}, \mathrm{DMSO}-d_{6}\right): \delta 167.7,167.3,134.9,134.6$, 132.6, 132.3, 130.0, 129.7, 128.4, 128.1; (Found: C, 54.71; H, 2.59; $\mathrm{N}$, 9.14. Cal for $\mathrm{C}_{14} \mathrm{H}_{8} \mathrm{Cl}_{2} \mathrm{~N}_{2} \mathrm{O}_{2}$ : C, 54.75; $\mathrm{H}, 2.63 ; \mathrm{N}, 9.12 \%$ ).

5.5.13. 2-(4-Chlorophenyl)-1H-benzo[d]imidazole-5-carboxylic Acid (6d). White solid; mp $194-196^{\circ} \mathrm{C}$; IR ( $\left.\mathrm{cm}^{-1}, \mathrm{KBr}\right)$ : 3090, 2821, 1907, 1676, 1624, 1425, 1319, 1026, 947, 835, 731; ${ }^{1} \mathrm{H}$ NMR (300 MHz, DMSO- $d_{6}$ ): $\delta 13.30$ (brs, $\left.1 \mathrm{H}\right), 12.77$ (brs, $1 \mathrm{H}), 8.19$ (m, 3H), 7.84 (brs, $1 \mathrm{H}), 7.65$ (m, 3H); ${ }^{13} \mathrm{C} \mathrm{NMR}$ $\left(75 \mathrm{MHz}, \mathrm{DMSO}-d_{6}\right): \delta 167.8,152.4,135.0,129.1,128.4,128.4$, 124.7, 123.8, 122.4, 119.9; (Found: C, 61.65; H, 3.31; N, 10.25. Cal for $\mathrm{C}_{14} \mathrm{H}_{9} \mathrm{ClN}_{2} \mathrm{O}_{2}$ : C, 61.66; H, 3.33; N, 10.27\%).

5.5.14. 2-(4-Nitrophenyl)-1H-benzo[d]imidazole-5-carboxylic Acid (6e). Yellow solid; mp $272-274^{\circ} \mathrm{C}$; IR $\left(\mathrm{cm}^{-1}, \mathrm{KBr}\right)$ : 3338, 2949, 1699, 1604, 1514, 1348, 1213, 853, 774; ${ }^{1} \mathrm{H}$ NMR $\left(300 \mathrm{MHz}, \mathrm{DMSO}-d_{6}\right.$ ) $): \delta 13.38(\mathrm{~s}, 2 \mathrm{H}), 8.39(\mathrm{~s}, 4 \mathrm{H}), 8.20(\mathrm{~s}$, $1 \mathrm{H}), 7.86(\mathrm{~d}, J=8.1 \mathrm{~Hz}, 1 \mathrm{H}), 7.69(\mathrm{~d}, J=8.1 \mathrm{~Hz}, 1 \mathrm{H}) ;{ }^{13} \mathrm{C} \mathrm{NMR}$ $\left(75 \mathrm{MHz}, \mathrm{DMSO}-d_{6}\right): \delta 168.0,151.5,148.4,142.3,139.5,135.4$, 131.8, 128.9, 128.0, 125.6, 124.5, 117.9, 115.3; (Found: C, 59.32; $\mathrm{H}, 3.15 ; \mathrm{N}, 14.81$. Cal for $\mathrm{C}_{14} \mathrm{H}_{9} \mathrm{~N}_{3} \mathrm{O}_{4}: \mathrm{C}, 59.37 ; \mathrm{H}, 3.20 ; \mathrm{N}$, $14.84 \%)$.

5.5.15. Phenyl(2-phenyl-1H-benzo[d]imidazol-5-yl)methanone (7a). Yellow solid; mp 221-222 ${ }^{\circ} \mathrm{C}$; IR ( $\left.\mathrm{cm}^{-1}, \mathrm{KBr}\right): 3375,3061$, $1645,1572,1321,902,707 ;{ }^{1} \mathrm{H}$ NMR $\left(300 \mathrm{MHz}, \mathrm{DMSO}-d_{6}\right)$ : $\delta 13.30(\mathrm{~s}, 1 \mathrm{H}), 8.20(\mathrm{~d}, J=6.2 \mathrm{~Hz}, 2 \mathrm{H}), 7.94$ (brs, $1 \mathrm{H}), 7.76-$ $7.54(\mathrm{~m}, 10 \mathrm{H}) ;{ }^{13} \mathrm{C}$ NMR $\left(75 \mathrm{MHz}, \mathrm{DMSO}_{-} d_{6}\right): \delta 195.5,138.0$, 132.0, 130.9, 130.4, 129.4, 129.0, 128.3, 126.6, 124.2; (Found: C, 80.43; H, 4.65; N, 9.31. Cal for $\mathrm{C}_{20} \mathrm{H}_{14} \mathrm{~N}_{2} \mathrm{O}$ : C, 80.52; H, 4.73; $\mathrm{N}, 9.39 \%)$.

5.5.16. 2-(2-Hydroxyphenyl)-1H-benzo[d]imidazol-5-yl)(phenyl)methanone (7b). Light yellow solid; mp 227-229 C; IR $\left(\mathrm{cm}^{-1}, \mathrm{KBr}\right): 3298,3057,1919,1726,1614,1450,1294,981,786$; ${ }^{1} \mathrm{H}$ NMR $\left(300 \mathrm{MHz}, \mathrm{DMSO}-d_{6}\right): \delta 12.91$ (brs, $\left.2 \mathrm{H}\right), 8.10$ (d, $J=$ $8.1 \mathrm{~Hz}, 1 \mathrm{H}), 8.08$ (brs, 1H), 7.81-7.75 (m, 4H), 7.69 (m, 1H), 7.58 (m, 2H), $7.42(\mathrm{~m}, 1 \mathrm{H}), 7.08-7.02(\mathrm{~m}, 2 \mathrm{H}) ;{ }^{13} \mathrm{C} \mathrm{NMR}(75 \mathrm{MHz}$, DMSO- $\left.d_{6}\right): \delta 196.5,158.0,154.3,138.3,133.0,132.8,131.9,130.0$, 129.0, 127.4, 135.3, 120.2, 117.7, 113.0; (Found: C, 76.41; H, 4.42; $\mathrm{N}$, 8.88. Cal for $\mathrm{C}_{20} \mathrm{H}_{14} \mathrm{~N}_{2} \mathrm{O}_{2}$ : C, 76.42; H, 4.49; N, 8.91\%).

5.5.17. 2-(2,6-Dichlorophenyl)-1H-benzo[d]imidazol-5-yl) (phenyl)methanone (7c). White solid; mp $164-166^{\circ} \mathrm{C}$; IR $\left(\mathrm{cm}^{-1}, \mathrm{KBr}\right): 3059,1734,1651,1431,1317,970,788 ;{ }^{1} \mathrm{H}$ NMR $\left(300 \mathrm{MHz}, \mathrm{DMSO}-d_{6}\right)$ : $\delta 13.29$ (brs, $\left.1 \mathrm{H}\right), 8.05$ (brs, $\left.1 \mathrm{H}\right)$, $7.74(\mathrm{~m}, 3 \mathrm{H}), 7.70-7.64(\mathrm{~m}, 5 \mathrm{H}), 7.58(\mathrm{t}, J=7.6 \mathrm{~Hz}, 2 \mathrm{H}) ;{ }^{13} \mathrm{C}$ NMR (75 MHz, DMSO- $\left.d_{6}\right): \delta 195.7,148.9,137.8,134.8,132.6$, 132.0, 129.9, 129.4, 128.3, 120.0; (Found: C, 65.38; H, 3.25; N, 7.70. Cal for $\mathrm{C}_{20} \mathrm{H}_{12} \mathrm{Cl}_{2} \mathrm{~N}_{2} \mathrm{O}$ : C, 65.41; H, 3.29; N, 7.63\%).

5.5.18. 2-(4-(1H-Benzo[d]imidazol-2-yl)phenyl)-1H-benzo[d] imidazole (8). White solid; $\mathrm{mp} 245-247^{\circ} \mathrm{C}$; IR $\left(\mathrm{cm}^{-1}, \mathrm{KBr}\right)$ : 3061, 1626, 1440, 1317, 1118, 966, 846, 740; ${ }^{1} \mathrm{H}$ NMR (300 MHz, DMSO- $\left.d_{6}\right): \delta 13.01$ (brs, $\left.2 \mathrm{H}\right), 8.33(\mathrm{~s}, 4 \mathrm{H}), 7.61(\mathrm{~s}, 4 \mathrm{H}), 7.23-$ $7.20(\mathrm{~m}, 4 \mathrm{H}) ;{ }^{13} \mathrm{C}$ NMR $\left(75 \mathrm{MHz}, \mathrm{DMSO}-d_{6}\right): \delta 150.3,138.9$, 130.7, 127.0, 122.6, 115.1; (Found: C, 77.31; H, 4.54; N, 17.98. Cal for $\mathrm{C}_{20} \mathrm{H}_{14} \mathrm{~N}_{4}$ : C, 77.40; $\mathrm{H}, 4.55 ; \mathrm{N}, 18.05 \%$ ).

\section{Acknowledgments}

The authors are grateful to Professor M. S. Wadia and Professor Dilip D. Dhavale for helpful discussions. Vaishali S. Shinde and Vyankat A. Sontakke are thankful to Department of Science and Technology (DST), New Delhi, for the financial support and Junior Research Fellowship (SR/S1/OC-89/ 2009), respectively. S. Ghosh thanks Council of Scientific and Industrial Research (CSIR), Government of India, for Senior Research Fellowship (09/137(0516)/2012-EMR-I).

\section{References}

[1] G. Balboni, C. Trapella, Y. Sasaki et al., "Influence of the side chain next to C-terminal benzimidazole in opioid pseudopeptides containing the Dmt-Tic pharmacophore," Journal of Medicinal Chemistry, vol. 52, no. 17, pp. 5556-5559, 2009.

[2] W. A. Maxwell and G. Brody, "Antifungal activity of selected benzimidazole compounds," Applied microbiology, vol. 21, no. 5, pp. 944-945, 1971.

[3] D. Sharma, B. Narasimhan, P. Kumar, and A. Jalbout, "Synthesis and QSAR evaluation of 2-(substituted phenyl)- $1 H$-benzimidazoles and [2-(substituted phenyl)-benzimidazol-1-yl]-pyridin3-yl-methanones,' European Journal of Medicinal Chemistry, vol. 44, no. 3, pp. 1119-1127, 2009.

[4] A. A. Farahat, E. Paliakov, A. Kumar et al., "Exploration of larger central ring linkers in furamidine analogues: synthesis and evaluation of their DNA binding, antiparasitic and fluorescence properties," Bioorganic \& Medicinal Chemistry, vol. 19, no. 7, pp. 2156-2167, 2011.

[5] D. Valdez-Padilla, S. Rodríguez-Morales, A. Hernández-Campos et al., "Synthesis and antiprotozoal activity of novel 1-methylbenzimidazole derivatives," Bioorganic \& Medicinal Chemistry, vol. 17, no. 4, pp. 1724-1730, 2009.

[6] Y. F. Li, G. F. Wang, P. L. He et al., "Synthesis and anti-hepatitis B virus activity of novel benzimidazole derivatives," Journal of Medicinal Chemistry, vol. 49, no. 15, pp. 4790-4794, 2006.

[7] H. Banie, A. Sinha, R. J. Thomas, J. C. Sircar, and M. L. Richards, "2-phenylimidazopyridines, a new series of golgi compounds with potent antiviral activity," Journal of Medicinal Chemistry, vol. 50, no. 24, pp. 5984-5993, 2007.

[8] P. S. Charifson, A. L. Grillot, T. H. Grossman et al., "Novel dual-targeting benzimidazole urea inhibitors of DNA gyrase and topoisomerase IV possessing potent antibacterial activity: intelligent design and evolution through the judicious use of structure-guided design and stucture-activity relationships," Journal of Medicinal Chemistry, vol. 51, no. 17, pp. 5243-5263, 2008. 
[9] J. Chen, Z. Wang, C. M. Li et al., "Discovery of novel 2-aryl4-benzoyl-imidazoles targeting the colchicines binding site in tubulin as potential anticancer agents," Journal of Medicinal Chemistry, vol. 53, no. 20, pp. 7414-7427, 2010.

[10] H. Göker, C. Kuş, D. W. Boykin, S. Yıldız, and N. Altanlar, "Synthesis of some new 2-substituted-phenyl-1H-benzimidazole-5carbonitriles and their potent activity against Candida species," Bioorganic \& Medicinal Chemistry, vol. 10, no. 8, pp. 2589-2596, 2002.

[11] G. N. Vázquez, L. Yépez, A. H. Campos et al., "Synthesis and antiparasitic activity of albendazole and mebendazole analogues," Bioorganic \& Medicinal Chemistry, vol. 11, no. 21, pp. 4615-4622, 2003.

[12] K. J. Spivak and Z. Amit, "Effects of pimozide on appetitive behavior and locomotor activity: dissimilarity of effects when compared to extinction," Physiology \& Behavior, vol. 36, no. 3, pp. 457-463, 1986.

[13] P. Lindberg, P. Nordberg, T. Alminger et al., "The mechanism of action of the gastric acid secretion inhibitor omeprazole," Journal of Medicinal Chemistry, vol. 29, no. 8, pp. 1327-1329, 1986.

[14] S. B. Mohan, T. P. Behera, and B. V. V. Ravi Kumar, "Microwave irradiation versus conventional method: synthesis of benzimidazolyl chalcone derivatives," International Journal of ChemTech Research, vol. 2, no. 3, pp. 1634-1637, 2010.

[15] A. K. Tiwari and A. Mishra, "Synthesis and antiviral activity of N-substituted-2-subastituted benzimidazole derivatives," Indian Journal of Chemistry B, vol. 45, pp. 489-493, 2006.

[16] R. J. Perry and B. D. Wilson, "A novel palladium-catalyzed synthesis of 2-arylbenzimidazoles," Journal of Organic Chemistry, vol. 58, no. 25, pp. 7016-7021, 1993.

[17] K. Bourgrin, A. Loupy, and M. Soufiaoui, "Trois nouvelles voies de synthèse des dérivés 1, 3-azoliques sous micro-ondes," Tetrahedron, vol. 54, no. 28, pp. 8055-8064, 1998.

[18] V. R. Ruiz, A. Corma, and M. J. Sabater, "New route for the synthesis of benzimidazoles by a one-pot multistep process with mono and bifunctional solid catalysts," Tetrahedron, vol. 66, no. 3, pp. 730-735, 2010.

[19] I. Bhatnagar and M. V. George, "Oxidation with metal oxides-II: oxidation of chalcone phenylhydrazones, pyrazolines, $o$-aminobenzylidine anils and $o$-hydroxy benzylidine anils with manganese dioxide," Tetrahedron, vol. 24, no. 3, pp. 1293-1298, 1968.

[20] P. L. Beaulieu, B. Haché, and E. Von Moos, "A practical oxonemediated, high-throughput, solution-phase synthesis of benzimidazoles from 1,2-phenylenediamines and aldehydes and its application to preparative scale synthesis," Synthesis, no. 11, pp. 1683-1692, 2003.

[21] M. A. Weidner-Wells, K. A. Ohemeng, V. N. Nguyen et al., "Amidino benzimidazole inhibitors of bacterial two-component systems," Bioorganic and Medicinal Chemistry Letters, vol. 11, no. 12, pp. 1545-1548, 2001.

[22] S. E. López, J. Restrepo, B. Pérez, S. Ortiz, and J. Salazar, "One pot microwave promoted synthesis of 2-aryl-1H-benzimidazoles using sodium hydrogen sulfite," Bulletin of the Korean Chemical Society, vol. 30, no. 7, pp. 1628-1630, 2009.

[23] P. Gogoi and D. Konwar, "An efficient and one-pot synthesis of imidazolines and benzimidazoles via anaerobic oxidation of carbon-nitrogen bonds in water," Tetrahedron Letters, vol. 47, no. 1, pp. 79-82, 2006.

[24] K. Bahrami, M. M. Khodaei, and F. Naali, "Mild and highly efficient method for the synthesis of 2-arylbenzimidazoles and 2-arylbenzothiazoles," Journal of Organic Chemistry, vol. 73, no. 17, pp. 6835-6837, 2008.

[25] M. A. Chari, D. Shobha, E. R. Kenawy, S. S. Al-Deyab, B. V. Subba Reddy, and A. Vinu, "Nanoporous aluminosilicate catalyst with 3D cage-type porous structure as an efficient catalyst for the synthesis of benzimidazole derivatives," Tetrahedron Letters, vol. 51, no. 39, pp. 5195-5199, 2010.

[26] C. Mukhopadhyay, S. Ghosh, S. Sengupta (Bandyopadhyay), and S. Deb, "Synthesis of 2-alkyl substituted benzimidazoles under microwave irradiation: anti-proliferative effect of some representative compounds on human histiocytic lymphoma cell U937," RSC Advances, vol. 1, no. 6, pp. 1033-1037, 2011.

[27] K. Wilson and J. H. Clark, "Solid acids and their use as environmentally friendly catalysts in organic synthesis," Pure and Applied Chemistry, vol. 72, no. 7, pp. 1313-1319, 2000.

[28] R. G. Jacob, L. G. Dutra, C. S. Radatz, S. R. Mendes, G. Perin, and E. J. Lenardão, "Synthesis of 1,2-disubstitued benzimidazoles using $\mathrm{SiO}_{2} / \mathrm{ZnCl}_{2}$," Tetrahedron Letters, vol. 50, no. 13, pp. 14951497, 2009.

[29] A. V. Patil, B. P. Bandgar, and S. H. Lee, "Silica supported fluoroboric acid: an efficient and reusable heterogeneous catalyst for facile synthesis of 2-aliphatic benzothiazoles, benzoxazoles, benzimidazoles and imidazo[4,5-b]pyridines," Bulletin of the Korean Chemical Society, vol. 31, no. 6, pp. 1719-1722, 2010.

[30] S. Paul and B. Basu, "Highly selective synthesis of libraries of 1, 2-disubstituted benzimidazoles using silica gel soaked with ferric sulfate," Tetrahedron Letters, vol. 53, no. 32, pp. 4130-4133, 2012.

[31] D. Kumar, D. N. Kommi, R. Chebolu, S. K. Garg, R. Kumar, and A. K. Chakraborti, "Selectivity control during the solid supported protic acids catalysed synthesis of 1,2-disubstituted benzimidazoles and mechanistic insight to rationalize selectivity," RSC Advances, vol. 3, pp. 91-98, 2013.

[32] S. Yamazaki, "Chromium(VI) oxide-catalyzed benzylic oxidation with periodic acid," Organic Letters, vol. 1, no. 13, pp. 21292132, 1999.

[33] L. Xu, J. Cheng, and M. L. Trudell, "Chromium(VI) oxide catalyzed oxidation of sulfides to sulfones with periodic acid," Journal of Organic Chemistry, vol. 68, no. 13, pp. 5388-5391, 2003.

[34] A. K. M. L. Rahman, M. Kumashiro, and T. Ishihara, "Direct synthesis of formic acid by partial oxidation of methane on $\mathrm{H}$ ZSM-5 solid acid catalyst," Catalysis Communications, vol. 12, no. 13, pp. 1198-1200, 2011.

[35] V. U. Pawar, S. Ghosh, B. A. Chopade, and V. S. Shinde, "Design and synthesis of harzialactone analogues: promising anticancer agents," Bioorganic and Medicinal Chemistry Letters, vol. 20, no. 24, pp. 7243-7245, 2010.

[36] M. Shen and T. G. Driver, "Iron(II) bromide-catalyzed synthesis of benzimidazoles from aryl azides," Organic Letters, vol. 10, no. 15, pp. 3367-3370, 2008. 

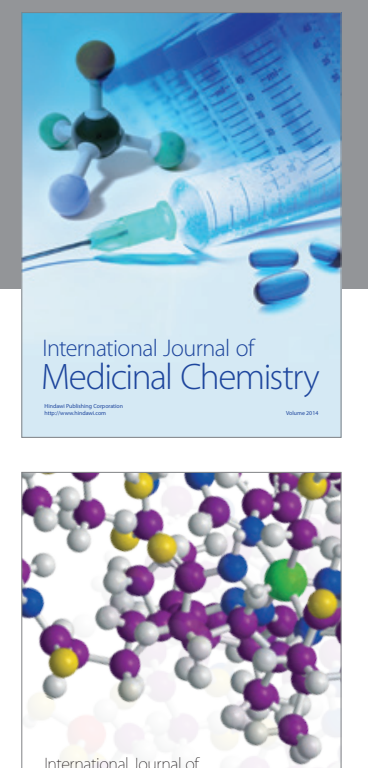

\section{Carbohydrate} Chemistry

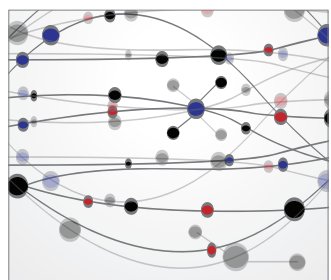

The Scientific World Journal
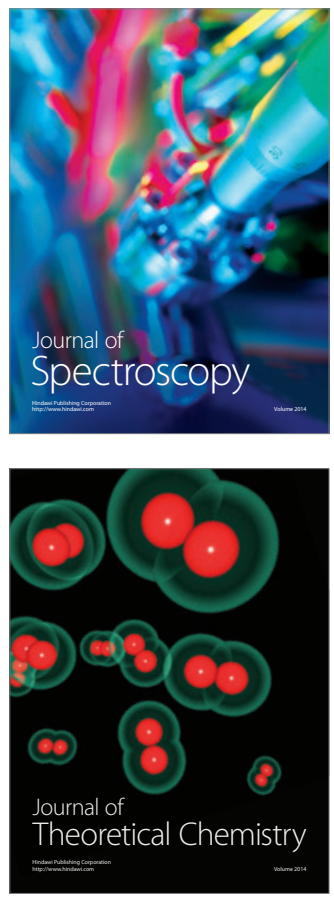
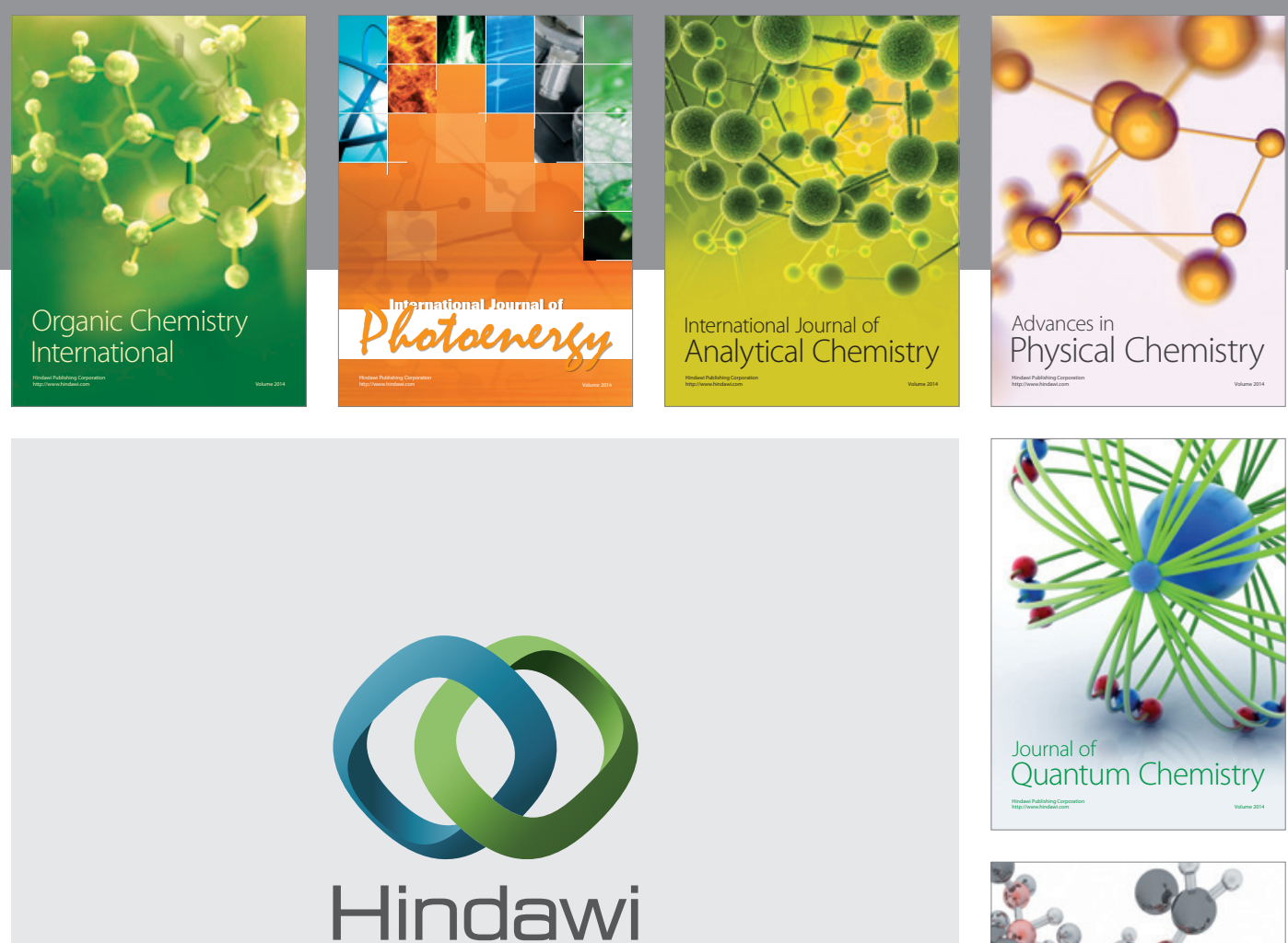

Submit your manuscripts at

http://www.hindawi.com

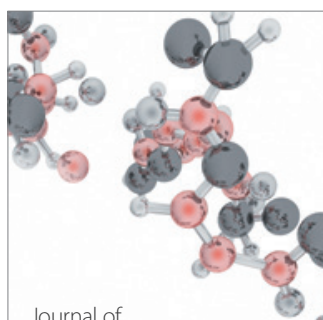

Analytical Methods

in Chemistry

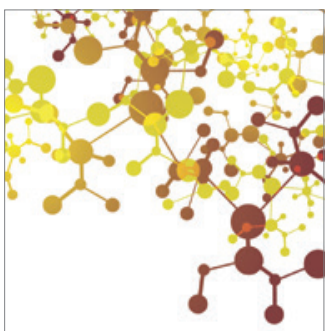

Journal of

Applied Chemistry

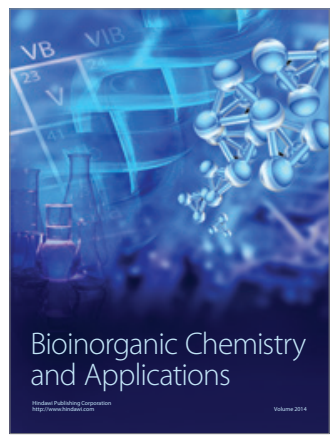

Inorganic Chemistry
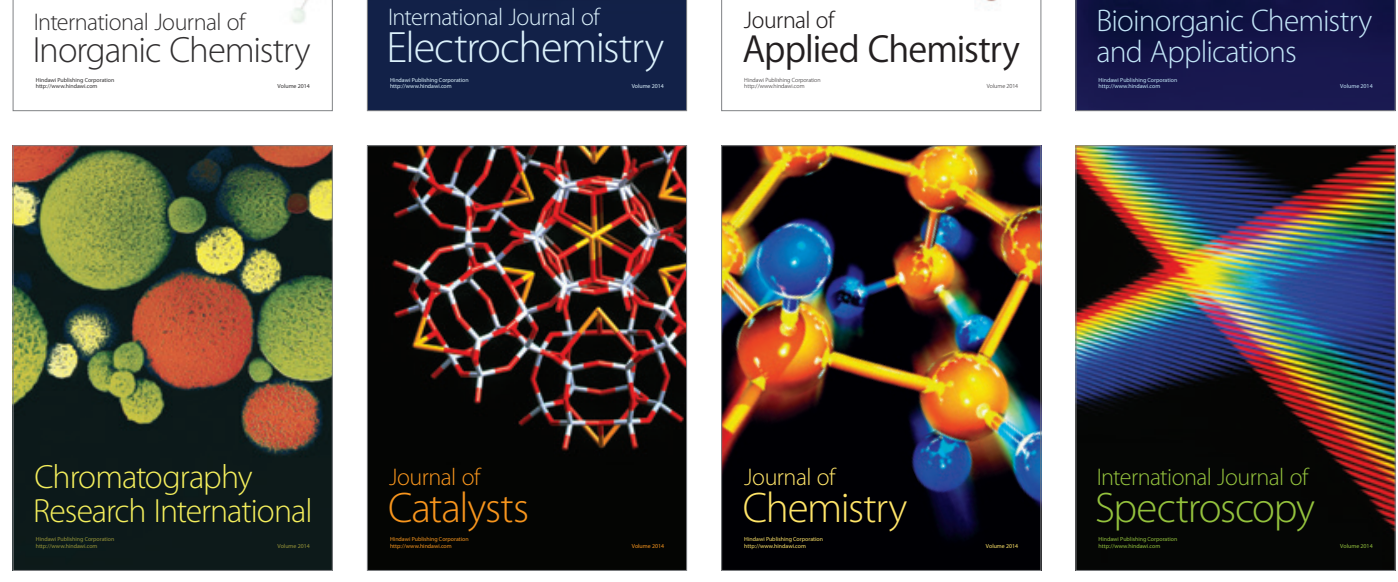